\title{
Transcription Factors for the Modulation of Pluripotency and Reprogramming
}

\author{
J.-C.D. HenG, ${ }^{1,2}$ Y.L. ORLOV, ${ }^{3}$ AND H.-H. NG ${ }^{1,2,4,5,6}$ \\ ${ }^{1}$ Gene Regulation Laboratory, Genome Institute of Singapore, Singapore 138672; ${ }^{2}$ NUS Graduate \\ School for Integrative Sciences and Engineering, Singapore 117597; ${ }^{3}$ Computational Systems Biology Group, \\ Genome Institute of Singapore, Singapore 138672; ${ }^{4}$ Department of Biochemistry, National University of Singapore, \\ Singapore 117597; ${ }^{5}$ Department of Biological Sciences, National University of Singapore, Singapore 117597; \\ ${ }^{6}$ School of Biological Sciences, Nanyang Technological University, Singapore 639798 \\ Correspondence: nghh@gis.a-star.edu.sg
}

\begin{abstract}
Pluripotency and self-renewal are the defining traits of embryonic stem cells (ESCs) and this status quo is maintained by the core transcription factors Oct4, Sox2, and Nanog. Genome-wide mapping of the binding sites of these pivotal factors and other ESC transcriptional regulators has unraveled the transcriptional network governing pluripotency. Strikingly, a sizeable fraction of the binding sites of Oct4 and Nanog are not conserved in mouse and human ESCs. Binding site turnover and the presence of species-specific transposable elements are some of the factors contributing to this disp arity. Hence, comparing human and mouse ESCs will shed new light on the design of transcriptional regulatory networks for pluripotency. Despite the significant differences among pluripotent mammalian stem cells, the same set of transcription factors (Oct4, Sox2, Klf4, and c-Myc) can be used to reprogram human and mouse somatic cells into induced pluripotent stem cells. Recent works also demonstrate that there are multiple ways of imparting pluripotency. For instance, the nuclear receptors Nr5a2 and Esrrb can, respectively, substitute for Oct4 and Klf4 in reprogramming. This chapter summarizes the different roles of transcription factors in the modulation of pluripotent states and in the induction of pluripotent phenotypes.
\end{abstract}

ESCs, first isolated from mouse preimplantation blastocysts almost 30 years ago (Evans and Kaufman 1981; Martin 1981), are known for their ability to self-renew indefinitely in vitro. These cells are considered pluripotent because they can differentiate into cells of all three embryonic germ layers: the endoderm, mesoderm, and ectoderm. As ESCs differentiate, developmental potency becomes more restricted and they become either specialized adult progenitor cells or terminally differentiated cells. Although ESCs contain the same genetic material as other cell types in the body, they can be maintained in vitro in a perpetual self-renewing state that is poised for differentiation. Different cell types possess different transcriptomes, and it is the unique transcriptome within ESCs that allows them to assume this distinct pluripotent and self-renewing identity. This variation in transcriptome that comprises all of the expressed transcripts within the cell can be accounted for by a host of factors. Principally, DNA-binding proteins known as transcription factors that interact with regulatory regions of genes such as promoters and enhancers can modulate the expression of genetic elements. Therefore, unraveling the transcriptional regulatory network in ESCs will provide invaluable insights into the biological network that preserves ESC identity. In addition, given their broad differentiation capacities, ESCs are good models to study the mechanisms underlying cellular differentiation. Furthermore, mouse ESCs are amenable to genetic modifications, and these cells can be used for the generation of transgenic mouse models.
Apart from murine ESCs, pluripotent stem cells have also been derived from human blastocysts (Thomson et al. 1998; Cowan et al. 2004). These human ESCs serve as an important cellular model to understand the pluripotency and self-renewing framework in the context of humans. In this regard, a comparative analysis of the transcriptional regulatory networks in both mouse and human ESCs will identify conserved and diverse regulatory mechanisms that govern ESC identity.

\section{PIVOTAL TRANSCRIPTION FACTORS IN ESCs: OCT4, SOX2, AND NANOG}

Oct 4 and Sox 2 are two transcription factors that are critical for the maintenance of the ESC state. When the expression of Oct4 is reduced, ESCs undergo differentiation into trophoblast-like cells (Nichols et al. 1998; Niwa et al. 2000). Similarly, the ablation of Sox 2 results in differentiation toward the trophectoderm lineage (Masui et al. 2007). The reduced expression of these transcription factors is detrimental to the preservation of ESC integrity, but their overexpression can also perturb the pluripotent and selfrenewing phenotypes of ESCs. A 50\% increment in Oct4 expression can result in differentiation of ESCs into cells of the primitive endoderm (Niwa et al. 2000), whereas Sox2 overexpression causes ESCs to differentiate to neuronal cells (Kopp et al. 2008). On the other hand, certain transcription factors are able to sustain pluripotency when they are overexpressed in ESCs. For example, Nanog was 
discovered through a genetic screen for factors that maintain ESCs in the absence of leukemia inhibitory factor (LIF) (Chambers et al. 2003). ESCs deficient in Nanog tend to exit the self-renewing state and undergo differentiation (Chambers et al. 2003; Mitsui et al. 2003; Chambers et al. 2007). However, the transcription factors Oct4, Sox2, and Nanog are not the only transcription factors that are important in the maintenance of ESCs because a large repertoire of transcription factors has also been discovered as modulators for the undifferentiated state of ESCs (Zhang et al. 2006; Lim et al. 2007; Cole et al. 2008; Dejosez et al. 2008; Tam et al. 2008; Yi et al. 2008).

The transcription factor trio of Oct4, Sox2 and Nanog forms the core of the ESC transcriptional regulator network that extends out to other transcription factors and epigenetic modifiers (Boyer et al. 2005; Loh et al. 2006). Homeostatic balance within this transcriptional network is maintained by the tight regulation of each factor that collectively sustains the self-renewal of ESCs while simultaneously poising them for differentiation. Hence, any perturbation to the ESC transcriptional network, especially to the transcriptional core, might induce a spontaneous loss of ESC identity.

\section{COMPREHENSIVE MAPPING OF TRANSCRIPTION FACTOR-BINDING SITES TO DECIPHER THE ESC TRANSCRIPTIONAL REGULATORY NETWORK}

To further decipher the transcriptional framework within ESCs, Kim et al. (2008) used an in vivo biotinylation ChIP (bioChIP)-chip technique to study the genomewide promoter-binding profiles of Oct4, Sox2, Klf4, c-Myc, Nanog, Dax1, Rex1, Nac1, and Zfp281, all of which are associated with either somatic cell reprogramming or pluripotency. Interestingly, the analyses of the global binding profiles of these nine transcription factors revealed that many downstream target genes were indeed cooccupied by several transcription factors. Strikingly, it was found that genes bound by at least five transcription factors have a tendency to be active in the ESC state. In contrast, genes that are bound by only a few transcription factors tend to be repressed in ESCs. Notably, these nine transcription factors can be further categorized into two distinct clusters based on their common genomic targets: Oct4, Sox2, Klf4, Nanog, Nac1, Zfp281, and Dax1 constitute one cluster, whereas Rex1 and c-Myc belong to a separate cluster.

In another independent study, ChIP-sequencing (ChIPseq), which couples ChIP technique with massively parallel DNA sequencing technology, was used to determine the genome-wide binding profiles of 13 transcription factors and two transcriptional coregulators in mouse ESCs (Chen et al. 2008). These include the key transcription factors associated with somatic cell reprogramming as well as transcription factors Stat3, Smad1, Zfx, n-Myc, Esrrb, Tcfcp211, and E2f1. Chen et al. (2008) observed a highdensity binding of multiple transcription factors to genomic targets that they termed multiple transcription factorbinding loci (MTL). Interestingly, the authors also found that the transcription factors form two major clusters: the Nanog-Oct4-Sox 2 cluster and the Myc-specific cluster. The Nanog-Oct4-Sox2 cluster is composed of Oct4, Sox2, Nanog, Stat3, and Smad1, whereas the Myc-specific cluster comprises c-Myc, n-Myc, Zfx, and E2f1. Transcription factors in each cluster tend to cooccupy their own and one another's genomic targets. Because Stat 3 and Smad1 are important downstream effectors of the LIF and BMP4 signaling pathways, respectively, this finding provides evidence for the integration of external signaling stemming from the cytokines that sustain mouse ESCs with the core transcriptional network of ESCs. Taken together, these genome-wide mapping studies have provided a global view of the transcriptional regulatory network in ESCs.

\section{SPECIES-SPECIFIC DIFFERENCES OF OCT4 AND NANOG BINDING, AND REWIRING OF THE TRANSCRIPTIONAL CIRCUITRY BY TRANSPOSABLE ELEMENTS}

Although substantial knowledge has been gathered about the transcriptional regulatory network in mouse ESCs, little is known about this network in human ESCs. Given the critical function of both Oct4 and Nanog in maintaining ESCs, the genome-wide binding profile of these key transcription factors in mouse and human ESCs would be expected to be highly similar. However, no extensive characterization and comparison of the binding of these factors between both species has been performed until very recently. Kunarso and coworkers generated ChIP-seq data sets for the genome-wide binding profiles of OCT4, NANOG, and CTCF in human ESCs and then compared them with existing ChIP-seq data sets generated from mouse ESCs (Chen et al. 2008; Kunarso et al. 2010). Strikingly, the OCT4 and NANOG binding in both mouse and human was found to be less conserved than previously thought. Among the most enriched OCT4- and NANOGbinding sites (top 10\%), only approximately $5 \%$ of these regions are homologously occupied in mouse ESCs. In contrast, based on the same statistical criteria, the insulator-binding transcription factor $\mathrm{CTCF}$, which has a relatively consistent binding pattern in different human cell types (Kim et al. 2007), had almost $50 \%$ of their binding sites conserved (Kunarso et al. 2010). These findings imply that the genome-wide binding landscape of pivotal ESC factors OCT4 and NANOG has changed significantly since the evolutionary divergence of both species, whereas other factors such as CTCF remained largely conserved.

Interestingly, some of the differences between the binding sites in both species have been attributed to the insertion of transposable elements, which were found to comprise up to $25 \%$ of the OCT4/NANOG-binding sites in both human and mouse. These sites that coincide with transposable elements were termed repeat associated binding sites (RABS). Strikingly, about $20 \%$ and $15 \%$ of the OCT4- and NANOG-binding regions, respectively, are RABS, whereas in mouse ESCs, about $7 \%$ and $17 \%$ of the Oct4- and Nanog-binding sites, respectively, are accounted for by RABS. The binding of OCT4 and NANOG at trans- 
posable elements suggests that the insertion of transposable elements might have rewired the transcriptional circuitry in pluripotent stem cells. However, despite the high occurrence of RABS in both species, barely $1 \%$ of the OCT 4 RABS are homologously bound in mouse. This is partly accounted for by the presence of species-specific transposable elements, given that a majority of the transposable element families exapted in both species are different.

Besides demonstrating the species-specific rewiring of the transcriptional circuitry by transposable elements, $\mathrm{Ku}-$ narso et al. (2010) also coupled the gene-binding data set with a gene-expression data set to identify the conserved and species-specific genes that were both bound and regulated by OCT4 and NANOG. By comparing the microarray data set of human ESCs subjected to POU5F1 RNA interference (RNAi) with the existing transcriptome data set of Pou5f1-knockdown mouse ESCs, they found that 137 of the orthologous genes were down-regulated in both mouse and human ESCs, and about half of these conserved targets possessed an OCT4/NANOG-binding site. Among these OCT4/NANOG-binding-site-containing conserved targets, $15 \%$ were homologously bound in both species, whereas the remainder exhibited a binding site turnover (Fig. 1). Binding site turnover is a phenomenon in which the binding site of a transcription factor, such as a promoter, is present in one species but absent in the other species. Instead, the latter has an alternative binding site not present in the former (Fig. 1). An example of a gene that displays binding site turnover is $A E B P 2$, whereas purely conserved targets include ESC-related genes such as POU5F1, SOX2, KLF4, and NANOG (Fig. 1). Besides elucidating the conserved targets, Kunarso et al. (2010) also narrowed down 584 genes that were down-regulated only in human ESCs and subsequently reported 50 humanspecific targets that possessed OCT4/NANOG-binding sites and were also associated with RABSs (Fig. 1).

Two recent studies have also reported the species-specific rewiring of the transcriptional regulatory networks in other models that include preimplantation embryos and liver cells (Schmidt et al. 2010; Xie et al. 2010). In the second study, Xie et al. performed global transcriptional profiling of embryos from three mammalian species at different stages of preimplantation embryonic development and coupled this data with ChIP-seq profiling of key transcriptional regulators (Xie et al. 2010). Their study revealed that maternally inherited transcripts in murine, bovine, and human embryos are more conserved than zygotically activated transcripts. Remarkably, the analyses also revealed that species-specific expression can be accounted for both by a modification of transcription factor binding sites resulting from single nucleotide mutations and the insertion of gene regulatory regions by transposons. These notable genomic changes are believed to have culminated in the murine-specific expression of genes such as $M t f 2$. In the
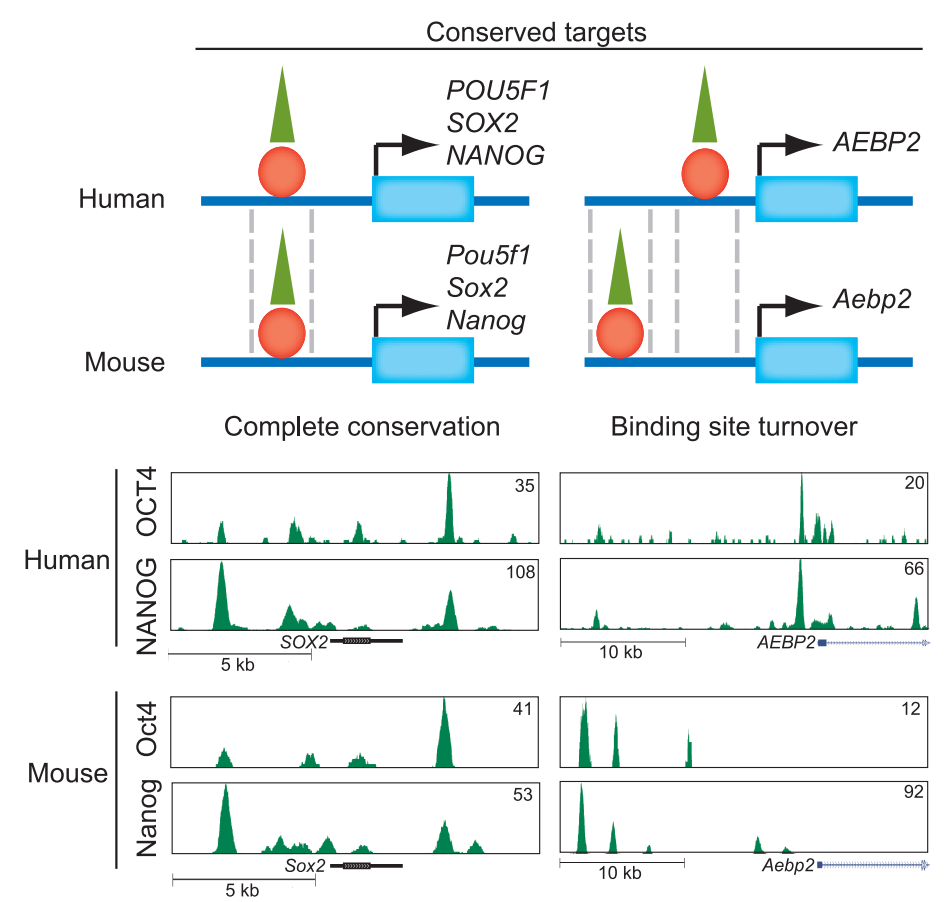
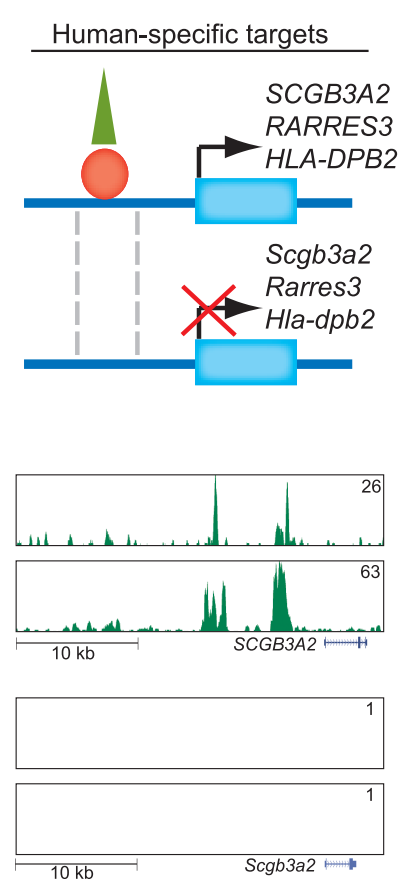

Figure 1. Conserved and species-specific regulation of genes by OCT4 and NANOG. Transcription factors (red circles) such as OCT4 and NANOG can bind at the regulatory regions such as gene promoters or enhancers in mouse and human ESCs. These genes are described as conserved targets. Genes such as POU5F1, SOX2, and NANOG have a very similar OCT4/NANOG-binding profile (green peaks) in both species (left panel). However, transcription factors may bind to a particular regulatory region in only one species, whereas the same transcription factor may bind to an alternative regulatory region in the other species. This occurrence is known as binding site turnover (middle panel). An example of a gene that exhibits binding site turnover is $A E B P 2$. In contrast to conserved targets, human-specific target genes such as $S C G B 3 A 2, R A R R E S 3$, and HLA-DPB2 are neither bound nor regulated by OCT4 and NANOG (right panel). 
first study, global binding of CEBP and HNF4A in the liver was compared among five different species, revealing extensive species-specific differences in transcription factor binding and gene regulation. Altogether, by comparing global transcription factor-binding profiles concomitantly with gene-expression analysis, these studies have provided new insights on the evolutionary rewiring of transcriptional regulatory networks.

It is intriguing that transposable elements might represent a new class of regulatory elements in the transcriptional regulatory network governing the pluripotency and self-renewal of ESCs. However, the role of transposable elements in the transcriptional control of ESCs has yet to be demonstrated. With a wealth of information about transcription factors that dictate pluripotency, it is now feasible to use transcription factors to mediate cell-fate changes. The ability to reprogram cells with transcription factors has now been added to a list of other reprogramming methodologies that include somatic cell nuclear transfer and cell fusion (Lewitzky and Yamanaka 2007).

\section{TRANSCRIPTION FACTOR REPROGRAMMING CODES FOR THE INDUCTION OF PLURIPOTENCY}

Despite the species-specific differences in the wiring of key transcription factors to the genome, certain ESC transcription factors can exert dominant effects on pluripotency-associated cellular identity in both mouse and human cells. In 2006, a groundbreaking study by Yamanaka and colleagues demonstrated the conversion of murine somatic cells to pluripotent cells by retroviral transduction of four transcription factors: Oct4, Sox2, Klf4, and c-Myc (Takahashi and Yamanaka 2006). These reprogrammed cells, also known as induced pluripotent stem cells (iPSCs), are highly similar to ESCs in terms of morphology, genetic expression, epigenetic marks, and their fulfillment of even the most stringent pluripotency assays (Takahashi and Yamanaka 2006; Okita et al. 2007;
Boland et al. 2009; Zhao et al. 2009). Interestingly, the same set of transcription factors could also induce a pluripotent phenotype in human somatic cells (Takahashi et al. 2007; Lowry et al. 2008; Park et al. 2008).

The ability of Oct 4 and Sox 2 to reprogram cells to pluripotency was not surprising given that these two transcription factors have been very well characterized in maintaining the self-renewal and pluripotency of ESCs (Nichols et al. 1998; Niwa et al. 2000; Masui et al. 2007). Similarly, c-Myc has also been implicated in the maintenance of ESCs, in which it was reported that the Myc protein works downstream from the LIF-Stat3 pathway (Cartwright et al. 2005). Intriguingly, Klf4 was an unexpected addition in the reprogramming cocktail because little was known about the Kruppel-like transcription factors in the context of ESCs. Nevertheless, subsequent to Yamanaka's finding, Ng and colleagues found that Klf4 as well as its close family members Klf2 and Klf5 are important in the self-renewal of mouse ESCs (Jiang et al. 2008). Another group (Ema et al. 2008) also showed that Klf5 is important for the maintenance of ESCs, further corroborating the findings by Jiang et al. (2008). A recent study (Hall et al. 2009) reported that Oct 4 and the LIF-Stat3 pathway activate Klf2 and Klf5, respectively, to support the self-renewal of ESCs. Although all three Klf proteins have been implicated in ESC self-renewal, there is in fact an observed redundancy among the three Klf proteins because only a triple knockdown of Klf2, Klf4, and Klf5 in mouse ESCs induced an overt differentiated phenotype (Jiang et al. 2008). In agreement with this finding, Nakagawa et al. (2008) reported that Klf2 and Klf5 could replace Klf4 in the reprogramming of somatic cells. Interestingly, besides the ability of close family members of Klf4, Sox2, and c-Myc to substitute their counterparts in reprogramming (Nakagawa et al. 2008), several Yamanaka factors can be replaced by other unrelated transcription factors (Fig. 2) (Feng et al. 2009; Heng et al. 2010). For instance, Esrrb, an orphan nuclear receptor, can replace Klf4 in the reprogramming of MEFs (Fig. 2) (Feng et al. 2009). More interestingly, another nuclear receptor,

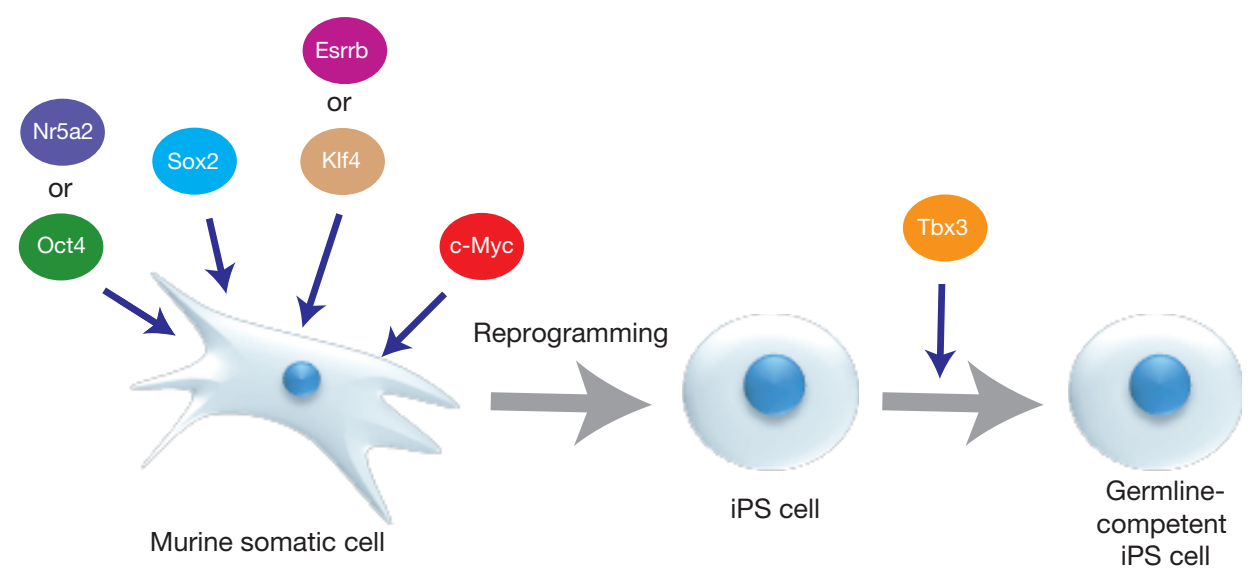

Figure 2. The role of transcription factors Nr5a2, Esrrb, and Tbx3 in reprogramming. Murine somatic cells can be reprogrammed to iPSCs with the defined cocktail of reprogramming factors comprising Oct4, Sox2, Klf4, and c-Myc (Takahashi and Yamanaka 2006). Interestingly, unrelated transcription factors can replace the Yamanaka factors in the conversion of somatic cells to pluripotent cells. For instance, the nuclear receptors Nr5a2 and Esrrb can replace the need for exogenous Oct4 and K1f4, respectively. Furthermore, Tbx3, a T-box factor, can improve the quality of iPSCs generated by enhancing the germline competency of iPSCs. 


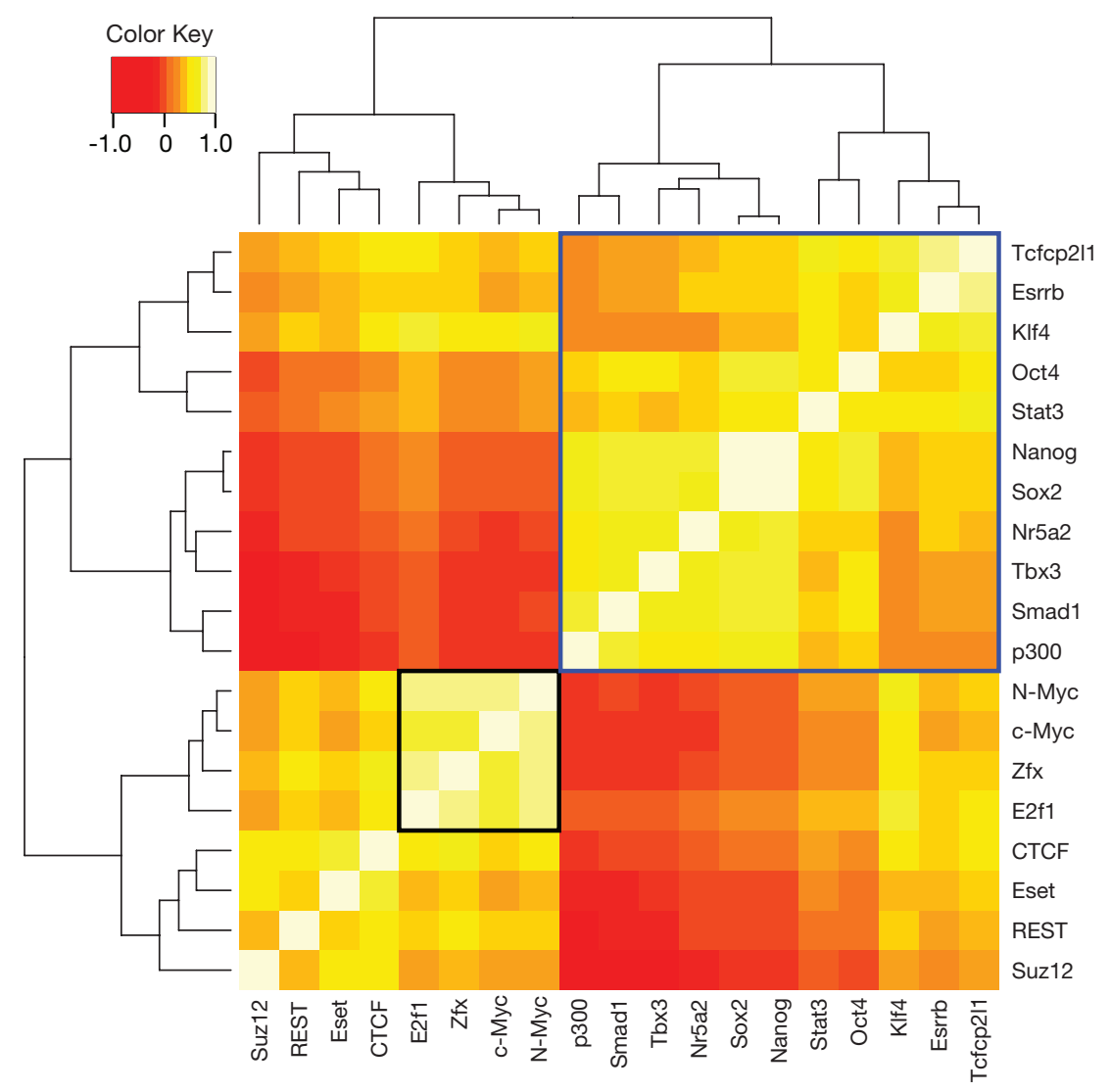

Figure 3. Reprogramming factors Nr5a2, Esrrb, and Tbx 3 cobind with the Nanog-Oct4-Sox 2 cluster of transcription factors. The heat map depicts the cooccurrence analysis of transcription factors within MTLs. The colocalization frequency of each pair of transcription factors is represented by the colors on the heat map in which yellow reflects a higher colocalization frequency and red reflects a lower colocalization frequency. Transcription factors are clustered along both axes according to their similarity in their cooccurrence with other factors. Whereas n-Myc, c-Myc, Zfx, and E2f1 form a distinct Myc-specific cluster (demarcated by the black box on the heat map), Nanog, Oct4, and Sox 2 form another separate cluster (demarcated by the blue box on heat map), which is also shared by Nr5a2, Esrrb, and Tbx3.

$\mathrm{Nr} 5 \mathrm{a} 2$, can replace exogenous Oct4 in the reprogramming of murine somatic cells (Fig. 2) (Heng et al. 2010). This finding adds new perspective to the reprogramming code for pluripotency because even Oct1 and Oct6, which are close family members of Oct 4 , are unable to substitute Oct4 in reprogramming (Nakagawa et al. 2008). Recently, the repertoire of transcription factors associated with reprogramming was further augmented with the discovery of Tbx3, a T-box factor, that could significantly improve the germline competency of murine iPSCs (Fig. 2) (Han et al. 2010).

Remarkably, the genome-wide binding analysis of $\mathrm{Nr} 5 \mathrm{a} 2$, Esrrb, and $\mathrm{Tbx} 3$, which were recently implicated in reprogramming, reveals that they tend to colocalize with factors within the Nanog-Oct4-Sox2 cluster (Fig. 3). This finding implies that these recently implicated reprogramming factors have significant roles in the maintenance of mouse ESCs. This notion is supported by a large-scale RNAi loss-of-function screen identifying Tbx 3 and Esrrb to be important in maintaining the self-renewal of mouse ESCs (Ivanova et al. 2006). In addition, it was previously shown that Esrrb is important in maintaining the pluripo- tency of mouse ESCs (Loh et al. 2006). The nuclear receptor $\mathrm{Nr} 5 \mathrm{a} 2$, which binds to the proximal promoter and proximal enhancer of the Pou5f1 gene (Gu et al. 2005), has also been implicated in the maintenance of mouse ESCs (Gu et al. 2005; Zhou et al. 2007; Tay et al. 2008).

Given the dual roles of transcription factors in the context of reprogramming and the maintenance of pluripotency in ESCs, our understanding of dedifferentiation and pluripotency shows that they should complement each other. However, it is noteworthy that the newly reported reprogramming factors Esrrb, Nr5a2, and Tbx3 have no reported roles in the maintenance of self-renewal and pluripotency in human ESCs. This could be due to species-specific differences of the transcriptional wiring in mouse and human ESCs.

\section{MODULATION OF PLURIPOTENT STATES BY TRANSCRIPTION FACTORS}

In addition to the species-specific transcriptional wiring of ESCs, other differences exist between mouse and human ESCs. For example, colony morphology and the 
growth requirements of mouse and human ESCs differ substantially. With respect to morphology, mouse ESCs grow as compact, dome-shaped colonies, whereas human ESCs grow as flat colonies. As for culture conditions, mouse ESCs require LIF and BMP4, whereas human ESCs are maintained in the presence of bFGF and activin/TGF- $\beta$. However, not all mouse pluripotent stem cell lines resemble mouse ESCs. Epiblast stem cells (EpiSCs) are stem cell lines derived from postimplantation mouse embryos (Brons et al. 2007; Tesar et al. 2007). Like ESCs, mouse EpiSCs are considered pluripotent because they can differentiate into multiple lineages in vitro and they can form teratomas when grafted into adult immunodeficient mice. However, in contrast to mouse ESCs, mouse EpiSCs have very limited potential to contribute to the generation of chimeras when introduced into early embryos, and these cells do not undergo germline transmission (Brons et al. 2007; Tesar et al. 2007). Hence, mouse ESCs and EpiSCs show differences in their developmental potentials in vivo. Interestingly, mouse EpiSCs resemble human ESCs in terms of morphology, X-chromosome inactivation in female cells, as well as its requirements for bFGF and activin signaling (Brons et al. 2007; Tesar et al. 2007). On this note, the different growth factor requirements and culture conditions for different pluripotent cells in different species suggest that alternative states of pluripotency do exist. The similarities between mouse EpiSCs and human ESCs have also led to the idea that human ESCs are the functional equivalents of mouse EpiSCs (Brons et al. 2007; Tesar et al. 2007).

Mouse EpiSCs are not locked in their specific pluripotent states but can be induced to transit from one state to another. Hence, these distinct pluripotent states are sometimes referred to as metastable states. Interestingly, EpiSCs could be converted to cells that resemble mouse ESCs by ectopic expression of Klf4 or Klf2 in the presence of LIF or by Nanog in either the presence or absence of LIF (Guo et al. 2009; Hall et al. 2009; Silva et al. 2009). These converted cells display an up-regulation of mouse ESC-specific genes as well as X-chromosome reactivation. However, in contrast to mouse ESCs, EpiSCs do not express Klf4 and express a lower level of Nanog (Brons et al. 2007; Tesar et al. 2007). Therefore, it is conceivable that together with appropriate culture conditions, the overexpression of genes that are highly expressed and are important in the maintenance of ESCs can also revert EpiSCs to mouse ESC-like cells. Moreover, because EpiSCs are pluripotent and are more plastic in differentiation potency than somatic cells, these cells can be reprogrammed to a ground-state level of pluripotency with only a single transcription factor (Guo et al. 2009; Silva et al. 2009) as compared to conventional reprogramming of somatic cells that requires the introduction of several factors (Takahashi and Yamanaka 2006; Takahashi et al. 2007; Yu et al. 2007). More interestingly, it was recently shown that EpiSCs could be converted to mouse ESC-like cells in the presence of LIF without any other exogenous factors (Bao et al. 2009). Nonetheless, the introduction of exogenous factors in the presence of LIF might still be required to further boost the efficiency of the generation of mouse ESC-like cells from EpiSCs.
Although human ESCs are highly similar to EpiSCs, the conversion of human ESCs to cells that resemble mouse ESCs might not be straightforward due to the inherent species-specific differences in their transcriptomes and epigenetic landscapes. Nonetheless, Li et al. (2009) first demonstrated such a conversion, in which they generated human iPSCs that resemble mESCs with respect to their morphologies by reprogramming human fibroblasts with Oct4, Sox2, Nanog, and Lin28 in mouse ESC media and human LIF. These colonies were then maintained in a cocktail of chemicals comprising inhibitors of GSK3 $\beta$, MEK, and Alk5. Apart from mouse ESC-like human iPSCs, human ESCs that resemble a mouse ESC-like state were also generated by several independent groups (Hanna et al. 2010; Xu et al. 2010). Remarkably, completely different approaches were adopted to derive human ESCs that resemble mouse ESCs. Ding and colleagues used culture conditions that consisted of LIF, MEK inhibitor, and a p38 inhibitor ( $\mathrm{Xu}$ et al. 2010), whereas Jaenisch and colleagues (Hanna et al. 2010) coupled the introduction of exogenous factors with chemical inhibitors that include a GSK3 $\beta$ inhibitor and a MEK inhibitor to generate mouse ESC-like human ESCs. Jaenisch and colleagues (Hanna et al. 2010) first embarked on the reprogramming of human somatic cells to human iPSCs that resemble mouse ESCs. Using a doxycycline-inducible system to express OCT4, SOX2, and KLF4 in secondary fibroblasts in the presence of GSK $3 \beta$ and MEK inhibitors as well as human LIF, they successfully converted human fibroblasts to human iPSCs. These human iPSCs were described as possessing a naïve state of pluripotency characterized by the absence of $\mathrm{X}$ inactivation in female cells. This naïve state of pluripotency is opposed to the primed state of pluripotency that is used to describe the pluripotent state of EpiSCs. It was shown that the constitutive expression of either Oct4 and Klf4 or Klf4 and Klf2 can maintain these naïve human iPSCs in the absence of druginduced expression of the reprogramming factors. Notably, the addition of Forskolin, a protein kinase A pathway agonist, can also dispense the need for the sustained expression of exogenous factors. Human ESCs could be converted to the naïve state by also introducing either OCT4 and KLF4 or KLF4 and KLF2 in the presence of the MEK inhibitor, GSK $3 \beta$ inhibitor, Forskolin, and LIF. The human iPSCs and ESCs generated in both studies appear to be dome shaped and have a faster growth rate. They can also be passaged as single cells using trypsin that will otherwise decrease viability if these cells were typical human ESCs that survive better when passaged as cell clumps. A third group derived mouse ESC-like human iPSCs without any chemical inhibitors, although the introduction of factors was still required (Buecker et al. 2010). Five factors (Oct4, Sox2, Klf4, c-Myc, and Nanog) were ectopically expressed using a drug-inducible system to reprogram human fibroblasts in the presence of human LIF (Buecker et al. 2010). However, when ectopic expression of the reprogramming factors was removed, these converted cells reverted to fibroblastic cells, thus indicating that these converted cells were largely dependent on the ectopic expression of factors and that their respective endogenous 
genes were not yet fully activated. Strikingly, these converted human iPSCs were shown to be more amenable to targeted genetic modifications such as homologous recombination (Buecker et al. 2010). Therefore, these cells may serve as a useful resource for the generation of transgenic human pluripotent stem cells in the future. Overall, there are different techniques that employ the use of exogenous factors and/or chemicals to derive mouse ESClike cells, which suggests that there are in fact multiple routes to transit from one pluripotent state to another.

It is interesting that defined transcription factors, besides having an integral role in the maintenance of pluripotency and self-renewal in ESCs, could also participate in various reprogramming processes such as the (1) reprogramming of somatic cells (Fig. 2), (2) reprogramming of EpiSCs to ground-state pluripotency, and (3) conversion of human ESCs/iPSCs to mouse ESC-like cells. Thus, the versatility of these transcription factors has allowed us to not only better understand the complex transcriptional framework in ESCs but also to rewire the transcriptional circuitry to initiate interconversion among the different pluripotent states.

\section{CONCLUSIONS}

Both mouse and human ESCs share the defining hallmarks of pluripotency and self-renewal. However, closer analysis of these cells has revealed that species-specific differences exist between them and these include variations in their transcriptome and global binding of transcription factors. Despite these marked distinctions between different pluripotent cell types, transcription factors that are pivotal in supporting the maintenance of ESCs can also have a role in allowing one pluripotent state to transit to another. It is noteworthy that these ESC-related factors have also been widely reported to mediate the dedifferentiation of somatic cells to a pluripotent state. Altogether, the wealth of information that has been acquired from the deciphering of the ESC transcription factor network will provide new insights into how transcription factors can mediate transcriptional resetting and programming of pluripotent cell fate.

\section{ACKNOWLEDGMENTS}

We thank Kevin Andrew Uy Gonzales and Felicia Huimei Hong for critical comments on the manuscript.

\section{REFERENCES}

Bao S, Tang F, Li X, Hayashi K, Gillich A, Lao K, Surani MA. 2009. Epigenetic reversion of post-implantation epiblast to pluripotent embryonic stem cells. Nature 461: 1292-1295.

Boland MJ, Hazen JL, Nazor KL, Rodriguez AR, Gifford W, Martin G, Kupriyanov S, Baldwin KK. 2009. Adult mice generated from induced pluripotent stem cells. Nature 461: 91-94.

Boyer LA, Lee TI, Cole MF, Johnstone SE, Levine SS, Zucker JP, Guenther MG, Kumar RM, Murray HL, Jenner RG, et al. 2005. Core transcriptional regulatory circuitry in human embryonic stem cells. Cell 122: 947-956.

Brons IG, Smithers LE, Trotter MW, Rugg-Gunn P, Sun B, Chuva de Sousa Lopes SM, Howlett SK, Clarkson A, Ahrlund-Richter L, Pedersen RA, Vallier L. 2007. Derivation of pluripotent epiblast stem cells from mammalian embryos. Nature 448: 191-195.

Buecker C, Chen HH, Polo JM, Daheron L, Bu L, Barakat TS, Okwieka P, Porter A, Gribnau J, Hochedlinger K, Geijsen N. 2010. A murine ESC-like state facilitates transgenesis and homologous recombination in human pluripotent stem cells. Cell Stem Cell 6: 535-546.

Cartwright P, McLean C, Sheppard A, Rivett D, Jones K, Dalton S. 2005. LIF/STAT3 controls ES cell self-renewal and pluripotency by a Myc-dependent mechanism. Development 132: 885896.

Chambers I, Colby D, Robertson M, Nichols J, Lee S, Tweedie S, Smith A. 2003. Functional expression cloning of Nanog, a pluripotency sustaining factor in embryonic stem cells. Cell 113: 643-655.

Chambers I, Silva J, Colby D, Nichols J, Nijmeijer B, Robertson M, Vrana J, Jones K, Grotewold L, Smith A. 2007. Nanog safeguards pluripotency and mediates germline development. $\mathrm{Na}$ ture 450: 1230-1234.

Chen X, Xu H, Yuan P, Fang F, Huss M, Vega VB, Wong E, Orlov YL, Zhang W, Jiang J, et al. 2008. Integration of external signaling pathways with the core transcriptional network in embryonic stem cells. Cell 133: 1106-1117.

Cole MF, Johnstone SE, Newman JJ, Kagey MH, Young RA. 2008. Tcf3 is an integral component of the core regulatory circuitry of embryonic stem cells. Genes Dev 22: 746-755.

Cowan CA, Klimanskaya I, McMahon J, Atienza J, Witmyer J, Zucker JP, Wang S, Morton CC, McMahon AP, Powers D, et al. 2004. Derivation of embryonic stem-cell lines from human blastocysts. N Engl J Med 350: 1353-1356.

Dejosez M, Krumenacker JS, Zitur LJ, Passeri M, Chu LF, Songyang Z, Thomson JA, Zwaka TP. 2008. Ronin is essential for embryogenesis and the pluripotency of mouse embryonic stem cells. Cell 133: 1162-1174.

Ema M, Mori D, Niwa H, Hasegawa Y, Yamanaka Y, Hitoshi S, Mimura J, Kawabe Y, Hosoya T, Morita M, et al. 2008. Kruppellike factor 5 is essential for blastocyst development and the normal self-renewal of mouse ESCs. Cell Stem Cell 3: 555-567.

Evans MJ, Kaufman MH. 1981. Establishment in culture of pluripotential cells from mouse embryos. Nature 292: 154-156.

Feng B, Jiang J, Kraus P, Ng JH, Heng JC, Chan YS, Yaw LP, Zhang W, Loh YH, Han J, et al. 2009. Reprogramming of fibroblasts into induced pluripotent stem cells with orphan nuclear receptor Esrrb. Nat Cell Biol 11: 197-203.

Gu P, Goodwin B, Chung AC, Xu X, Wheeler DA, Price RR, Galardi C, Peng L, Latour AM, Koller BH, et al. 2005. Orphan nuclear receptor LRH-1 is required to maintain Oct4 expression at the epiblast stage of embryonic development. Mol Cell Biol 25: 3492-3505.

Guo G, Yang J, Nichols J, Hall JS, Eyres I, Mansfield W, Smith A. 2009. Klf4 reverts developmentally programmed restriction of ground state pluripotency. Development 136: 1063-1069.

Hall J, Guo G, Wray J, Eyres I, Nichols J, Grotewold L, Morfopoulou S, Humphreys P, Mansfield W, Walker R, et al. 2009. Oct4 and LIF/Stat3 additively induce Kruppel factors to sustain embryonic stem cell self-renewal. Cell Stem Cell 5: 597-609.

Han J, Yuan P, Yang H, Zhang J, Soh BS, Li P, Lim SL, Cao S, Tay J, Orlov YL, et al. 2010. Tbx3 improves the germ-line competency of induced pluripotent stem cells. Nature 463: 10961100 .

Hanna J, Cheng AW, Saha K, Kim J, Lengner CJ, Soldner F, Cassady JP, Muffat J, Carey BW, Jaenisch R. 2010. Human embryonic stem cells with biological and epigenetic characteristics similar to those of mouse ESCs. Proc Natl Acad Sci 107: 9222 9227.

Heng JC, Feng B, Han J, Jiang J, Kraus P, Ng JH, Orlov YL, Huss M, Yang L, Lufkin T, et al. 2010. The nuclear receptor Nr5a2 can replace Oct4 in the reprogramming of murine somatic cells to pluripotent cells. Cell Stem Cell 6: 167-174.

Ivanova N, Dobrin R, Lu R, Kotenko I, Levorse J, DeCoste C, Schafer X, Lun Y, Lemischka IR. 2006. Dissecting self-renewal in stem cells with RNA interference. Nature 442: 533-538.

Jiang J, Chan YS, Loh YH, Cai J, Tong GQ, Lim CA, Robson P, Zhong S, Ng HH. 2008. A core Klf circuitry regulates self-re- 
newal of embryonic stem cells. Nat Cell Biol 10: 353-360.

Kim TH, Abdullaev ZK, Smith AD, Ching KA, Loukinov DI, Green RD, Zhang MQ, Lobanenkov VV, Ren B. 2007. Analysis of the vertebrate insulator protein CTCF-binding sites in the human genome. Cell 128: 1231-1245.

Kim J, Chu J, Shen X, Wang J, Orkin SH. 2008. An extended transcriptional network for pluripotency of embryonic stem cells. Cell 132: 1049-1061.

Kopp JL, Ormsbee BD, Desler M, Rizzino A. 2008. Small increases in the level of Sox 2 trigger the differentiation of mouse embryonic stem cells. Stem Cells 26: 903-911.

Kunarso G, Chia NY, Jeyakani J, Hwang C, Lu X, Chan YS, Ng HH, Bourque G. 2010. Transposable elements have rewired the core regulatory network of human embryonic stem cells. Nat Genet 42: 631-634.

Lewitzky M, Yamanaka S. 2007. Reprogramming somatic cells towards pluripotency by defined factors. Curr Opin Biotechnol 18: $467-473$.

Li W, Wei W, Zhu S, Zhu J, Shi Y, Lin T, Hao E, Hayek A, Deng H, Ding S. 2009. Generation of rat and human induced pluripotent stem cells by combining genetic reprogramming and chemical inhibitors. Cell Stem Cell 4: 16-19.

Lim LS, Loh YH, Zhang W, Li Y, Chen X, Wang Y, Bakre M, Ng HH, Stanton LW. 2007. Zic3 is required for maintenance of pluripotency in embryonic stem cells. Mol Biol Cell 18: 1348 1358.

Loh YH, Wu Q, Chew JL, Vega VB, Zhang W, Chen X, Bourque G, George J, Leong B, Liu J, et al. 2006. The Oct4 and Nanog transcription network regulates pluripotency in mouse embryonic stem cells. Nat Genet 38: 431-440.

Lowry WE, Richter L, Yachechko R, Pyle AD, Tchieu J, Sridharan R, Clark AT, Plath K. 2008. Generation of human induced pluripotent stem cells from dermal fibroblasts. Proc Natl Acad Sci 105: 2883-2888.

Martin GR. 1981. Isolation of a pluripotent cell line from early mouse embryos cultured in medium conditioned by teratocarcinoma stem cells. Proc Natl Acad Sci 78: 7634-7638.

Masui S, Nakatake Y, Toyooka Y, Shimosato D, Yagi R, Takahashi K, Okochi H, Okuda A, Matoba R, Sharov AA, et al. 2007. Pluripotency governed by Sox 2 via regulation of Oct3/4 expression in mouse embryonic stem cells. Nat Cell Biol 9: 625-635.

Mitsui K, Tokuzawa Y, Itoh H, Segawa K, Murakami M, Takahashi K, Maruyama M, Maeda M, Yamanaka S. 2003. The homeoprotein Nanog is required for maintenance of pluripotency in mouse epiblast and ES cells. Cell 113: 631-642.

Nakagawa M, Koyanagi M, Tanabe K, Takahashi K, Ichisaka T, Aoi T, Okita K, Mochiduki Y, Takizawa N, Yamanaka S. 2008. Generation of induced pluripotent stem cells without Myc from mouse and human fibroblasts. Nat Biotechnol 26: 101-106.

Nichols J, Zevnik B, Anastassiadis K, Niwa H, Klewe-Nebenius D, Chambers I, Scholer H, Smith A. 1998. Formation of pluripotent stem cells in the mammalian embryo depends on the POU transcription factor Oct4. Cell 95: 379-391.

Niwa H, Miyazaki J, Smith AG. 2000. Quantitative expression of Oct-3/4 defines differentiation, dedifferentiation or self-renewal of ES cells. Nat Genet 24: 372-376.

Okita K, Ichisaka T, Yamanaka S. 2007. Generation of germlinecompetent induced pluripotent stem cells. Nature 448: 313-317.

Park IH, Zhao R, West JA, Yabuuchi A, Huo H, Ince TA, Lerou PH, Lensch MW, Daley GQ. 2008. Reprogramming of human somatic cells to pluripotency with defined factors. Nature 451: 141-146.

Schmidt D, Wilson MD, Ballester B, Schwalie PC, Brown GD, Marshall A, Kutter C, Watt S, Martinez-Jimenez CP, Mackay S, et al. 2010. Five-vertebrate ChIP-seq reveals the evolutionary dynamics of transcription factor binding. Science 328: 10361040.

Silva J, Nichols J, Theunissen TW, Guo G, van Oosten AL, Barrandon O, Wray J, Yamanaka S, Chambers I, Smith A. 2009. Nanog is the gateway to the pluripotent ground state. Cell 138: 722-737.

Takahashi K, Yamanaka S. 2006. Induction of pluripotent stem cells from mouse embryonic and adult fibroblast cultures by defined factors. Cell 126: 663-676.

Takahashi K, Tanabe K, Ohnuki M, Narita M, Ichisaka T, Tomoda $\mathrm{K}$, Yamanaka S. 2007. Induction of pluripotent stem cells from adult human fibroblasts by defined factors. Cell 131: 861-872.

Tam WL, Lim CY, Han J, Zhang J, Ang YS, Ng HH, Yang H, Lim B. 2008. T-cell factor 3 regulates embryonic stem cell pluripotency and self-renewal by the transcriptional control of multiple lineage pathways. Stem Cells 26: 2019-2031.

Tay YM, Tam WL, Ang YS, Gaughwin PM, Yang H, Wang W, Liu R, George J, Ng HH, Perera RJ, et al. 2008. MicroRNA-134 modulates the differentiation of mouse embryonic stem cells, where it causes post-transcriptional attenuation of Nanog and LRH1. Stem Cells 26: 17-29.

Tesar PJ, Chenoweth JG, Brook FA, Davies TJ, Evans EP, Mack DL, Gardner RL, McKay RD. 2007. New cell lines from mouse epiblast share defining features with human embryonic stem cells. Nature 448: 196-199.

Thomson JA, Itskovitz-Eldor J, Shapiro SS, Waknitz MA, Swiergiel JJ, Marshall VS, Jones JM. 1998. Embryonic stem cell lines derived from human blastocysts. Science 282: 11451147.

Xie D, Chen CC, Ptaszek LM, Xiao S, Cao X, Fang F, Ng HH, Lewin HA, Cowan C, Zhong S. 2010. Rewirable gene regulatory networks in the preimplantation embryonic development of three mammalian species. Genome Res 20: 804-815.

Xu Y, Zhu X, Hahm HS, Wei W, Hao E, Hayek A, Ding S. 2010. Revealing a core signaling regulatory mechanism for pluripotent stem cell survival and self-renewal by small molecules. Proc Natl Acad Sci 107: 8129-8134.

Yi F, Pereira L, Merrill BJ. 2008. Tcf3 functions as a steady-state limiter of transcriptional programs of mouse embryonic stem cell self-renewal. Stem Cells 26: 1951-1960.

Yu J, Vodyanik MA, Smuga-Otto K, Antosiewicz-Bourget J, Frane JL, Tian S, Nie J, Jonsdottir GA, Ruotti V, Stewart R, et al. 2007. Induced pluripotent stem cell lines derived from human somatic cells. Science 318: 1917-1920.

Zhang J, Tam WL, Tong GQ, Wu Q, Chan HY, Soh BS, Lou Y, Yang J, Ma Y, Chai L, et al. 2006. Sall4 modulates embryonic stem cell pluripotency and early embryonic development by the transcriptional regulation of Pou5f1. Nat Cell Biol 8: 11141123.

Zhao XY, Li W, Lv Z, Liu L, Tong M, Hai T, Hao J, Guo CL, Ma QW, Wang L, et al. 2009. iPS cells produce viable mice through tetraploid complementation. Nature 461: 86-90.

Zhou Q, Chipperfield H, Melton DA, Wong WH. 2007. A gene regulatory network in mouse embryonic stem cells. Proc Natl Acad Sci 104: 16438-16443. 


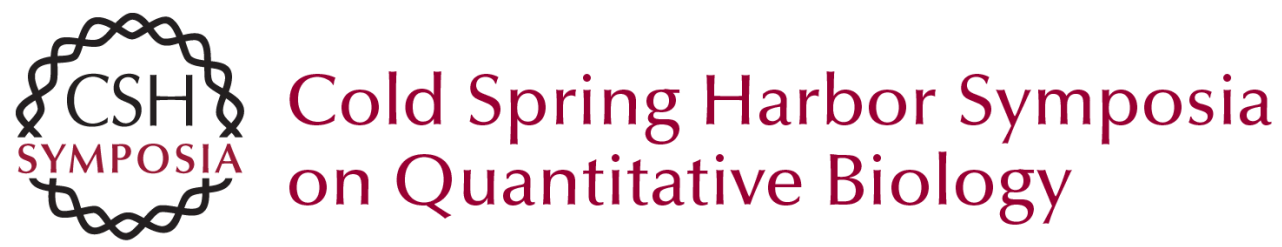

\section{Transcription Factors for the Modulation of Pluripotency and Reprogramming}

J.-C.D. Heng, Y.L. Orlov and H.-H. Ng

Cold Spring Harb Symp Quant Biol 2010 75: 237-244 originally published online November 3, 2010 Access the most recent version at doi:10.1101/sqb.2010.75.003

References This article cites 55 articles, 14 of which can be accessed free at: http://symposium.cshlp.org/content/75/237.full.html\#ref-list-1

\section{License}

Email Alerting Receive free email alerts when new articles cite this article - sign up in the box at the Service top right corner of the article or click here. 